\title{
Reduction of a single granule drying model: An essential step in preparation of a population balance model with a continuous growth term
}

Mortier, Séverine Thérèse F.C.; Daele, Timothy, Van; Gernaey, Krist V.; Beer, Thomas, De; Nopens, Ingmar

Published in:

A I Ch E Journal

Link to article, DOI:

10.1002/aic. 13907

Publication date:

2013

Document Version

Publisher's PDF, also known as Version of record

Link back to DTU Orbit

Citation (APA):

Mortier, S. T. F. C., Daele, T. V., Gernaey, K. V., Beer, T. D., \& Nopens, I. (2013). Reduction of a single granule drying model: An essential step in preparation of a population balance model with a continuous growth term. $A$ I Ch E Journal, 59(4), 1127-1138. https://doi.org/10.1002/aic.13907

\section{General rights}

Copyright and moral rights for the publications made accessible in the public portal are retained by the authors and/or other copyright owners and it is a condition of accessing publications that users recognise and abide by the legal requirements associated with these rights.

- Users may download and print one copy of any publication from the public portal for the purpose of private study or research.

- You may not further distribute the material or use it for any profit-making activity or commercial gain

- You may freely distribute the URL identifying the publication in the public portal 


\title{
Reduction of a Single Granule Drying Model: an Essential Step in Preparation of a Population Balance Model with a Continuous Growth Term
}

\author{
Séverine Thérèse F.C. Mortier \\ BIOMATH, Dept. of Mathematical Modelling, Statistics and Bioinformatics, Faculty of Bioscience Engineering, \\ Ghent University, 9000 Ghent, Belgium \\ Laboratory of Pharmaceutical Process Analytical Technology, Dept. of Pharmaceutical Analysis, Faculty of \\ Pharmaceutical Sciences, Ghent University, 9000 Ghent, Belgium \\ Timothy Van Daele \\ BIOMATH, Dept. of Mathematical Modelling, Statistics and Bioinformatics, Faculty of Bioscience Engineering, \\ Ghent University, 9000 Ghent, Belgium \\ Krist V. Gernaey \\ Center for Process Engineering and Technology, Dept. of Chemical and Biochemical Engineering, Technical \\ University of Denmark, 2800 Kongens Lyngby, Denmark \\ Thomas De Beer* \\ Laboratory of Pharmaceutical Process Analytical Technology, Dept. of Pharmaceutical Analysis, Faculty of \\ Pharmaceutical Sciences, Ghent University, 9000 Ghent, Belgium \\ Ingmar Nopens \\ BIOMATH, Dept. of Mathematical Modelling, Statistics and Bioinformatics, Faculty of Bioscience Engineering, \\ Ghent University, 9000 Ghent, Belgium
}

DOI 10.1002/aic.13907

Published online September 11, 2012 in Wiley Online Library (wileyonlinelibrary.com).

The development of a Population Balance Model (PBM) for a pharmaceutical granule drying process requires a continuous growth term; the latter actually represents the drying process as the moisture content is the internal coordinate of the PBM. To establish such a PBM, a complex drying model for a single granule needs reduction in complexity. The starting point is a detailed model that describes the drying behavior of single pharmaceutical granules. A Global Sensitivity Analysis (GSA) was performed to detect the most sensitive degrees of freedom in the model as these need to be retained in the reduced model. Simulations of the complex drying model were, in a next phase, used to develop the reduced model, which describes the decrease of the moisture content in function of the gas temperature. The developed reduced model was then included in a Population Balance Equation (PBE) to describe the drying behavior of a population of granules. () 2012 American Institute of Chemical Engineers AIChE J, 59: 1127-1138, 2013

Keywords: drying, pharmaceuticals, mathematical modeling, model reduction, PBM

\section{Introduction}

\section{Production of pharmaceutical tablets and its modeling}

The production of tablets consists of several consecutive steps. After blending of the individual components, a granulation step is often performed to agglomerate the particles into granules. The incorporation of a wet granulation operation in the tabletting production process requires the drying of the wet granules afterward. A fluidized bed drying process

\footnotetext{
*Shared last authorship.

Correspondence concerning this article should be addressed to I. Nopens at ingmar.nopens@ugent.be.

(C) 2012 American Institute of Chemical Engineers
}

is a commonly used technique for drying in pharmaceutical applications. The drying process might influence several properties of the granules and, hence, the further downstream processing. ${ }^{1,2}$

Mechanistic modeling of pharmaceutical processes has proven to be very useful for the pharmaceutical industry, also because nowadays there is a trend to move toward continuous production processes. ${ }^{2}$ To fully take advantage of continuous processes in terms of quality assurance, these processes have to rely on on-line measurements and realtime adjustment of controllable input variables. Understanding the process in detail (i.e., how it reacts to changes in controllable inputs) can be achieved by developing a 
calibrated and validated mechanistic model of the process. Indeed, the mechanistic model can be analyzed to compare the relative importance and specific impact of the different parameters on each model output. Such information can be used to force the process to stay inside the Design Space. ${ }^{3}$ To model the entire production process of tablets, all process steps have to be modeled separately. Here, the focus will only rely on the drying process.

The development of a complete mechanistic model for the fluidized bed drying process was discussed by Mortier et al. ${ }^{3}$ It is a tedious task and requires a step-wise approach. A drying model to describe the evolution of the moisture content of one single pharmaceutical granule is used as the starting point. This model can then be extended toward a population of granules using Population Balance Model (PBM). The interaction with the spatial component can further be studied using Computational Fluid Dynamics (CFD) and its integration with PBM (CFD-PBM). ${ }^{3}$ Specifically for a drying operation, the latter combination should allow to describe the spatial distribution of the moisture content of the granules in the dryer.

In the frame of this research, a mechanistic model is developed for a six-segmented fluidized bed drying system, which is part of a fully continuous from-powder-to-tablet manufacturing line (ConsiGma $^{\mathrm{TM}}$, Collette ${ }^{\mathrm{TM}}$, GEA Pharma Systems). A thoroughly calibrated and validated drying model that describes the drying behavior of single pharmaceutical granules in the six-segmented fluidized bed drying system at different gas temperatures has been presented earlier. ${ }^{2}$ The model developed in this article allows the description of the drying behavior of a population of wet granules as a function of the temperature of the drying gas. The incentive for developing such a model will be explained later.

\section{Introduction to $P B M$}

An often made assumption when modeling the system behavior of a population of individuals is that the system can be described by the "averaged" behavior of these individuals. However, this assumption might not be valid (and is most probably not) in a real system, as both spatial and population heterogeneity occur, meaning that the "state" of individual particles can be different and also the environment they are exposed to is not the same throughout the drying unit. This is likely to have an impact on the system behavior, although it is not known how significant this might be and, hence, needs to be explored. For a more detailed analysis of particles, interacting with each other and the continuous phase and, hence, the impact of these heterogeneities on the system behavior, PBMs can be applied. ${ }^{3}$

The general Population Balance Equation (PBE) describing the change of the number density distribution $n(x, r, t)$ is given by

$$
\begin{aligned}
\frac{\partial}{\partial t} n(x, r, t)+\nabla \dot{X}(x, r, Y, t) n(x, r, t) & \\
& +\nabla \dot{R}(x, r, Y, t) n(x, r, t)=h(x, r, Y, t)
\end{aligned}
$$

where $x, r, t, Y$, and $h$ are the internal coordinate (i.e., the internal property of the particles that is considered to be distributed), the external (spatial) coordinate vector, the time, the continuous phase vector, and the net birth rate due to discrete processes like, for example, aggregation or breakup. $\nabla$, the nabla operator, is a vector differential operator. In a three-dimensional Cartesian coordinate system, $\nabla$ is defined in terms of partial derivative operators as

$$
\nabla=\hat{x} \frac{\partial}{\partial x}+\hat{y} \frac{\partial}{\partial y}+\hat{z} \frac{\partial}{\partial z}
$$

where $\hat{x}, \hat{y}$, and $\hat{z}$ represent the unit vectors in each spatial direction. $\dot{X}$ and $\dot{R}$ are the partial derivatives of the internal and external coordinates, respectively. ${ }^{4}$ Drying of wet granules is a continuous process and, when assuming constant ambient conditions (i.e., no spatial variation), the general PBE can be reduced to

$$
\frac{\partial}{\partial t} n\left(R_{\mathrm{w}}, t\right)+\frac{\partial}{\partial R_{\mathrm{w}}} \dot{R_{\mathrm{w}}}\left(R_{\mathrm{w}}, Y\right) n\left(R_{\mathrm{w}}, t\right)=0
$$

where $R_{\mathrm{w}}$ is the wet radius of the particle, which is chosen as the internal coordinate. In this equation, the growth term is responsible for the continuous decrease of the moisture content.

$$
G_{\mathrm{r}}=\dot{R_{\mathrm{w}}}\left(R_{\mathrm{w}}, Y\right)
$$

with $G_{\mathrm{r}}$ the growth term. Here, $Y$ refers to the ambient conditions that the individual particles experience, which in this case can be the gas temperature, the gas velocity, the humidity of the air, and, as so forth these are all relevant variables that can interfere with the drying process. Equations 3 and 4 are according to the authors defined here for the first time for a pharmaceutical drying process and are, hence, innovative.

In the literature, population balances with a continuous growth term have mainly been formulated and used in the area of crystallization processes, where the size of the crystals is the internal coordinate of interest. There, the growth term is a positive term. ${ }^{5}$ A growth term dependent on the internal coordinate is referred to as a size-dependent growth term. Solution methods for this type of PBE have been described in the literature. ${ }^{6-8}$

The structure of Eq. 4 for a continuous drying process is, however, quite complex. ${ }^{2}$ It cannot as such be implemented in Eq. 3 and needs to be reduced by means of a model reduction step. An overview of techniques for that purpose is given first.

\section{Short review on model reduction techniques}

Model reduction techniques are usually described in the literature for very complex models, for example, semiconductor devices, weather forecast models, molecular systems, and so forth. ${ }^{9}$ Such detailed complicated physically based mathematical models are time consuming to solve and require the use of sophisticated hardware and software resources. ${ }^{10}$

A first group of model reduction techniques are heuristic model reduction methods. However, these require a lot of user input. A detailed analysis of the model behavior with respect to the selected set of parameters is needed. The interaction and feedback between model components to identify key processes of the system should be assessed. The changes in model structure must be decided upon by domain experts. ${ }^{11}$

Another group of model reduction techniques are those based on mathematical concepts. Several methods are projection based, that is, where the systems are projected onto a 
lower-dimensional subspace and the model equations are solved for the substituted projected states. ${ }^{12}$ A class in this area is based on Singular Value Decomposition (SVD), which cannot be applied to highly complex systems. For nonlinear systems the Proper Orthogonal Decomposition (POD) methods (also known as Principal Component Analysis (PCA)) is one of the possibilities. ${ }^{9}$ This method was applied by Banerjee et al. ${ }^{10}$ for a Rapid Thermal Processing (RTP) system. ${ }^{10}$ The eigenfunctions from the POD method are subsequently used as basis function in spectral Galerkin expansions of the governing partial differential equations solved by the finite element method to generate the reduced models. A good agreement between the reduced model and the original model was obtained, and a reduction in execution time was found.

Krylov-based approximation methods are another class of methods within the projection-based methods and can be implemented iteratively. As such, this method can be applied to systems of high complexity. ${ }^{9}$ Antoulas combined the SVD-based and Krylov-based methods, which is appropriate for application to large-scale circuits arising in Very-LargeScale Integration (VLSI) chip performance verification. ${ }^{9}$

The most simple nonlinear model reduction methods are based on linearization or reduced-order series expansion of the system's nonlinearities using Taylor or Volterra series. However, these methods are only applicable for weakly nonlinear systems. ${ }^{13}$

Bernhardt $^{12}$ described a data adaptive model reduction scheme, which can be applied to the transformation and reduction of systems of Ordinary Differential Equations (ODEs). It is a multistep approach using a low-dimensional projection of the model data followed by a Genetic Programming/Genetic Algorithm hybrid method to evolve the new model systems. ${ }^{12}$ POD and parameter tuning importance are two techniques used and compared by Degenring et al. ${ }^{14}$ The PCA can be used as a self-controlled routine, which means that the procedure can be repeated automatically until a predefined upper-limit of the error-functionals is achieved, which is advantageous over the parameter tuning importance technique. The latter should be used step-by-step, and each model reduction step should be studied critically.

Reduction methods based on evaluating the sensitivity of the performance indicators to a parameter vector are also known. The Advanced Rate Elimination Method (AREM) belongs to this category and focuses on the importance of individual rates, leading to a reduction of the number of rates. The Variable Simplification Method (VSM) looks at the importance of variation of each state variable to indicate which variable can be set to a constant value. No prior detailed understanding about the model is required. Both methods have been used to reduce ecosystem models, which helped to understand the mechanisms that influence ecosystem health indicators. ${ }^{15}$

In this work, the model reduction is performed on the full drying model of a single granule. A custom model reduction procedure is introduced, and the reduced model is implemented and demonstrated in the PBM model for description of the drying of a population of granules.

\section{Materials and Methods}

\section{Drying Model}

The drying model under study consists of two submodels each describing a distinct drying phase. The first drying phase entails the evaporation of water from the droplet free surface. The evaporation rate in this phase is given by

$$
\dot{m}_{\mathrm{v}, 1}=h_{\mathrm{D}}\left(\rho_{\mathrm{v}, \mathrm{s}}-\rho_{\mathrm{v}, \infty}\right) A_{\mathrm{d}}
$$

where $\dot{m}_{\mathrm{v}}$ is the mass transfer rate of the first drying phase, $h_{\mathrm{D}}$, the mass transfer coefficient, $\rho_{\mathrm{v}, \mathrm{s}}$, the partial vapor density near the droplet surface, $\rho_{\mathrm{v}, \infty}$, the partial vapor density in the ambient air, and $A_{\mathrm{d}}$, the surface area of the droplet. When the radius of the droplet equals the radius of the dry particle, the second drying phase starts, in which two regions are formed: a wet core and a dry crust. In the second phase, the evaporation rate is given by

$$
\begin{aligned}
\dot{m}_{\mathrm{v}, 2}=-\frac{8 \pi \epsilon^{4912.4 * e^{-0.024282 * T_{\mathrm{g}}}} D_{\mathrm{v}, \mathrm{cr}} M_{\mathrm{w}} p_{\mathrm{g}}}{\Re\left(T_{\mathrm{cr}, \mathrm{s}}+T_{\mathrm{wc}, \mathrm{s}}\right)} \\
\times \ln \left[\frac{p_{\mathrm{g}}-p_{\mathrm{v}, \mathrm{i}}}{p_{\mathrm{g}}-\left(\frac{\Re}{4 \pi M_{\mathrm{w}} h_{\mathrm{D}} R_{\mathrm{p}}^{2}} \dot{m}_{\mathrm{v}}+\frac{p_{\mathrm{v}, \infty}}{T_{\mathrm{g}}}\right) T_{\mathrm{p}, \mathrm{s}}}\right]
\end{aligned}
$$

with $\dot{m}_{\mathrm{v}, 2}$ the mass transfer rate of the second drying phase, $\epsilon$ the crust porosity, $D_{\mathrm{v}, \mathrm{cr}}$ the vapor diffusion coefficient (crust pores), $M_{\mathrm{w}}$ the molecular weight of the liquid, $p_{\mathrm{g}}$ the pressure of the drying agent, $T_{\mathrm{cr}, \mathrm{s}}$ and $T_{\mathrm{wc}, \mathrm{s}}$, respectively, the temperature of the crust outer surface and of the crust-wet core interface, $p_{\mathrm{v}, \mathrm{i}}$ and $p_{\mathrm{v}, \infty}$, respectively the partial vapor pressure at the crust-wet core interface and in the ambient air, $h_{\mathrm{D}}$ the mass transfer coefficient, $R_{\mathrm{p}}$ the particle radius, and $T_{\mathrm{g}}$ the temperature of the drying agent. The vapor, evaporated at the interface between the wet core and the dry crust, diffuses through the crust pores until it exits the pores, and it forms a thin boundary layer over the particle surface. This vapor is removed through advection by the air flow. The complete drying model is extensively described by Mortier et al. ${ }^{2}$ who also calibrated and validated it with experimental data.

\section{Global Sensitivity Analysis}

A Global Sensitivity Analysis (GSA) is performed to detect the most sensitive degrees of freedom in the drying model. GSA is the study of how the uncertainty in the output of a model (numerical or otherwise) can be apportioned to different sources of uncertainty in the model input (or model structure, parameters). ${ }^{16}$ This provides useful information if one is to reduce the model output uncertainty. A GSA can be carried out by a Monte Carlo procedure, after which a linear regression is performed. The result is based on repeated random sampling from probability density functions that are defined for each source of uncertainty.

The GSA in this study was performed using five degrees of freedom $(r)$ : the gas temperature, the gas velocity $\left(V_{\mathrm{g}}\right)$, humidity of the gas $(\mathrm{RH})$, the pressure of the gas, and the initial temperature of the particle $\left(T_{\mathrm{p}, 0}\right)$. These five degrees of freedom were specifically chosen on the basis of their sensitivity (evidenced earlier in Ref. 2) and the ability to adapt or control these degrees of freedom during the operation of the dryer. Indeed, the gas velocity can be set in the fluidized bed dryer (ConsiGma ${ }^{\mathrm{TM}}$ ). The humidity of the inlet air can be measured and controlled using specialized equipment. The initial temperature depends on the temperature set point of the granulator. The pressure at the inlet is also measured. All other degrees of freedom, in the drying model are fixed and cannot be controlled. For each degree of freedom an uncertainty range was determined based on physical 
Table 1. Range of the Degrees of Freedom Used in the Monte Carlo Simulation

\begin{tabular}{lc}
\hline Parameter & Range \\
\hline$T_{\mathrm{g}}\left({ }^{\circ} \mathrm{C}\right)$ & $20-80$ \\
$V_{\mathrm{g}}\left(\mathrm{m}^{3} / \mathrm{h}\right)$ & $150-500$ \\
$\mathrm{RH}(\%)$ & $1-15$ \\
$p_{\mathrm{g}}(\mathrm{Pa})$ & $100,500-101,700$ \\
$T_{\mathrm{p}, 0}\left({ }^{\circ} \mathrm{C}\right)$ & $25-50$ \\
\hline
\end{tabular}

limitations and physical reality. As no prior knowledge on values for the degrees of freedom is available, the degrees of freedom are sampled from a uniform distribution between chosen minimum and maximum values (the uncertainty range). The range of the degrees of freedom was based on the physical boundaries of the fluidized bed dryer for which the mechanistic model is developed and is given in Table 1.

The Latin Hypercube Sampling method was used for the generation of a probabilistic sampling of the degrees of freedom space, proposed by McKay et al. ${ }^{17}$ It is assumed that no correlation occurred between the different parameters. In total, 2000 samples were generated ( $M$ is the size of the Monte Carlo experiment). The matrix of the degrees of freedom is denoted by $Z$. In this matrix, the rows correspond to a combination of degrees of freedom fed into the drying model for one simulation

$$
Z=\left(\begin{array}{ccccc}
T_{\mathrm{g}, 1} & V_{\mathrm{g}, 1} & \mathrm{RH}_{1} & p_{\mathrm{g}, 1} & T_{\mathrm{p}, 0,1} \\
T_{\mathrm{g}, 2} & V_{\mathrm{g}, 2} & \mathrm{RH}_{2} & p_{\mathrm{g}, 2} & T_{\mathrm{p}, 0,2} \\
& & \cdots & & \\
T_{\mathrm{g}, N} & V_{\mathrm{g}, M} & \mathrm{RH}_{N} & p_{\mathrm{g}, M} & T_{\mathrm{p}, 0, M}
\end{array}\right)
$$

The evaluation of the sensitivity analysis is performed for both drying phases separately. As such, the sensitivity of the model to the degrees of freedom could be evaluated for both periods separately and can be different. The output variable used in the GSA is the evolution of the granule's water content, which for the drying model corresponds, respectively, to the decrease of the radius of the particle (first drying phase) and the decrease of the wet core radius (second drying phase) as a function of time. The time step used in the simulation is, respectively, 0.02 and $0.2 \mathrm{~s}$ for the first and the second drying phase.

If one assumes that the model has an error-free linear form, the following is valid ${ }^{18}$

$$
Y=\sum_{j=1}^{r} \Omega_{j} Z_{j}
$$

with $Y$ the output of interest, $\Omega_{j}$ the fixed coefficients, and $Z_{j}$ the independent variables that are normally distributed $\left[Z_{j} \sim\right.$ $N\left(\bar{z}_{j}, \sigma_{Z_{j}}\right)$ with $\bar{z}_{j}=0$ for $j=1,2 \ldots r$ (where $\bar{z}_{j}$ and $\sigma_{Z_{j}}$ are, respectively, the mean and the standard deviation of the degree of freedom)]. Additional assumptions are

$$
\begin{gathered}
\sigma_{Z_{1}}<\sigma_{Z_{2}}<\ldots<\sigma_{Z_{\mathrm{r}}} \\
\Omega_{1}>\Omega_{2}>\ldots>\Omega_{\mathrm{r}}
\end{gathered}
$$

The assumption mentioned in Eq. 9 is due to Eq. 8. Because the independent variables are normally distributed, the output variable $Y$ is also normally distributed, and the standard deviation of the output $\sigma_{Y}$ becomes

$$
\sigma_{Y}=\sqrt{\sum_{j=1}^{r} \Omega_{j}^{2} \sigma_{Z_{j}}^{2}}
$$

If the relative importance of $Z_{j}$ on the output variable $Y$ is of interest, the partial derivative of $Y$ to $Z_{j}$ is most often taken

$$
S_{Z_{j}}^{d}=\frac{\partial Y}{\partial Z_{j}}
$$

which yields for the linear model $S_{Z j}^{d}=\Omega_{j}$. However, this is not really reasonable. Because this means that the ordering of the factors by importance would be

$$
Z_{1}>Z_{2}>\ldots>Z_{\mathrm{r}}
$$

Equation 11 can be improved by normalizing the derivative by the input-output standard deviations

$$
S_{Z_{j}}^{\sigma}=\frac{\sigma_{Z_{j}} \partial Y}{\sigma_{Y} \partial Z_{j}}=\Omega_{j} \frac{\sigma_{Z_{j}}}{\sigma_{Y}}
$$

If Eq. 13 is combined with Eq. 10, then it can be concluded that

$$
\sum_{j=1}^{r}\left(S_{Z_{j}}^{\sigma}\right)^{2}=1
$$

The use of the normalized equation is a good way to rank the different input factors based on sensitivity. It depends both on $\sigma$ and $\Omega$, just as it should, and second the sensitivity measures are normalized to one.

This is the reason why after performing a Monte Carlo simulation, the output $(Y)$ at a specific point in time of the simulation is processed using a linear regression. ${ }^{19}$ This linear regression is performed on the scaled output and scaled degrees of freedom (autoscaling: scaling by first subtracting the mean followed by division by the standard deviation).

$$
Y_{(i)}=b_{0}+\sum_{j=1}^{r} b_{Z_{j}} Z_{j}^{(i)}
$$

with $Y_{(i)}$ the output for one simulation (considering the growth for drying periods 1 and 2 separately), $Z_{j}^{(i)}$ are the degrees of freedom used in this simulation, and $b_{0}$ and $b_{Z_{j}}$ are, respectively, the intercept and linear coefficients of the linear model that is constructed. The coefficients $b_{0}$ and $b_{Z_{j}}$ are determined by solving a least squares problem, based on the squared differences between the output values produced by the regression model and the actual model output produced by Monte Carlo simulation. Asymptotically $\hat{b_{0}} \cong 0$ and $\hat{b_{Z_{j}}} \cong \Omega_{j}$ for $j=1,2 \ldots r$. Besides these coefficients, their standardized equivalents $\hat{\beta}_{Z_{j}}$ (the Standardized Regression Coefficient (SRC) are determined as

$$
\hat{\beta}_{Z_{j}}=\hat{b}_{Z_{j}} \sigma_{Z_{j}} / \sigma_{Y} \cong \Omega_{j} \sigma_{Z_{j}} / \sigma_{Y}
$$

Comparing Eq. 16 with Eq. 13, it can be proven that $\hat{\beta}_{Z_{j}}$ coincides with $S_{Z j}^{\sigma}$ for linear models. Therefore, for linear models

$$
\sum_{j=1}^{r}\left(S_{Z_{j}}^{\sigma}\right)^{2}=\sum_{j=1}^{r}\left(\hat{\beta}_{Z_{j}}\right)^{2}=1
$$




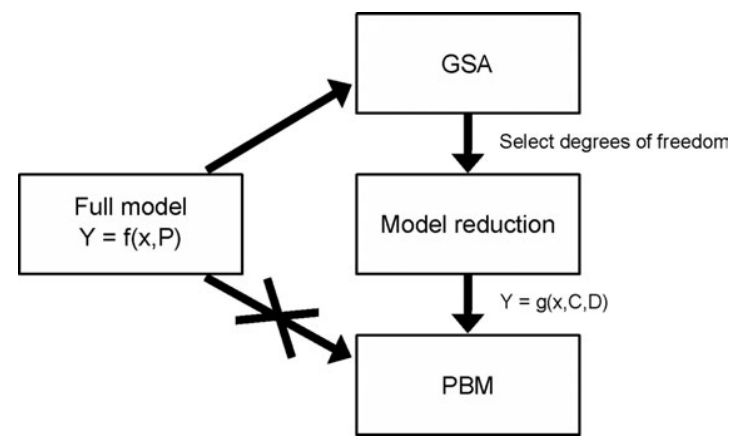

Figure 1. Problem statement.

The full model is too complex to be incorporated in the PBM directly, and therefore GSA is applied in the frame of achieving a reduced model that can be incorporated in the PBM.

If the model is nonlinear, both measures $\left(\hat{\beta}_{Z_{j}}\right.$ and $\left.S_{Z j}^{\sigma}\right)$ will be different, but the $\beta$ s will be a more robust and reliable measure of sensitivity, even for nonlinear models. $\beta$ s take the entire space of input factors into account, which is advantageous over $S_{Z j}^{\sigma}$. To ensure a reliable value for $\beta, M$ should be large compared to $r$. The $\sum_{j=1}^{r}\left(\hat{\beta}_{Z_{j}}\right)^{2}$ equals the fraction of linearity of the model, more precisely known as the coefficient of determination, $R_{Y}^{2}$, which is equal to the fraction of variance of the original data (Monte Carlo simulation results), explained by the regression model (Eq. 15). ${ }^{19}$ This value should at least be 0.7 for SRC to be a valid technique.

\section{Solution method for the PBE}

The PBE is solved using a High Resolution Finite Volume (HRFV) scheme. ${ }^{6}$ The high-resolution schemes were developed for compressible fluid dynamics, resolve sharp peaks, and shock discontinuities on coarse grids. Less numerical diffusion and the avoidance of numerical dispersion are the advantages. The improved numerical accuracy enables to use a coarser grid and to perform long-term simulations. The scheme is discrete in space but continuous in time, and the resulting system of ODEs can be solved by an ODE solver. More details about the solution method can be found in Qamar et al. ${ }^{6}$ In this work, a value of $1 / 3$ for $\kappa$ and the flux limiting function of Koren were used.

\section{Results \\ Procedure}

Evaluating the different available methods for model reduction, described in the "Introduction" section, it was concluded that none of the techniques based on mathematical concepts was feasible for our specific case. Antoulas ${ }^{9}$ used the model reduction techniques on a set of $n$ coupled first-order ODEs to replace them with $k$ coupled first-order ODEs where $k \ll n{ }^{9}$ The reduction of second-order systems remained an open problem. Chahlaoui et al. ${ }^{20}$ used a secondorder balanced truncation to reduce a second-order linear time-invariant system. Stability, error bounds, choice of Gramians (set of vectors) remained a problem. ${ }^{20}$ In fact for all projection-based methods, nonlinear model reduction is difficult. ${ }^{12}$ The ability to use the reduction method described by Bernhardt $^{12}$ depends on the system dynamics being confined to a low-dimensional subspace. The method is applied for simple models with oscillatory dynamics. Several reduction methods are quite complex to use. Van Nes et al. ${ }^{11}$ formulated "The most drastic way to simplify the model is to make an independent minimal model that describes the dominant mechanisms of the full model." This approach is very powerful, if both models produce qualitatively similar results. ${ }^{21}$ A new strategy was developed here. Basically, it involves a GSA step and a model reduction step (Figure 1). A GSA is performed on the full model $(f)$ to detect the most sensitive degrees of freedom. The full model is function of variables $(x)$ and parameters $(P)$. Second, the outcome of the sensitivity analysis will be used to develop an empirical

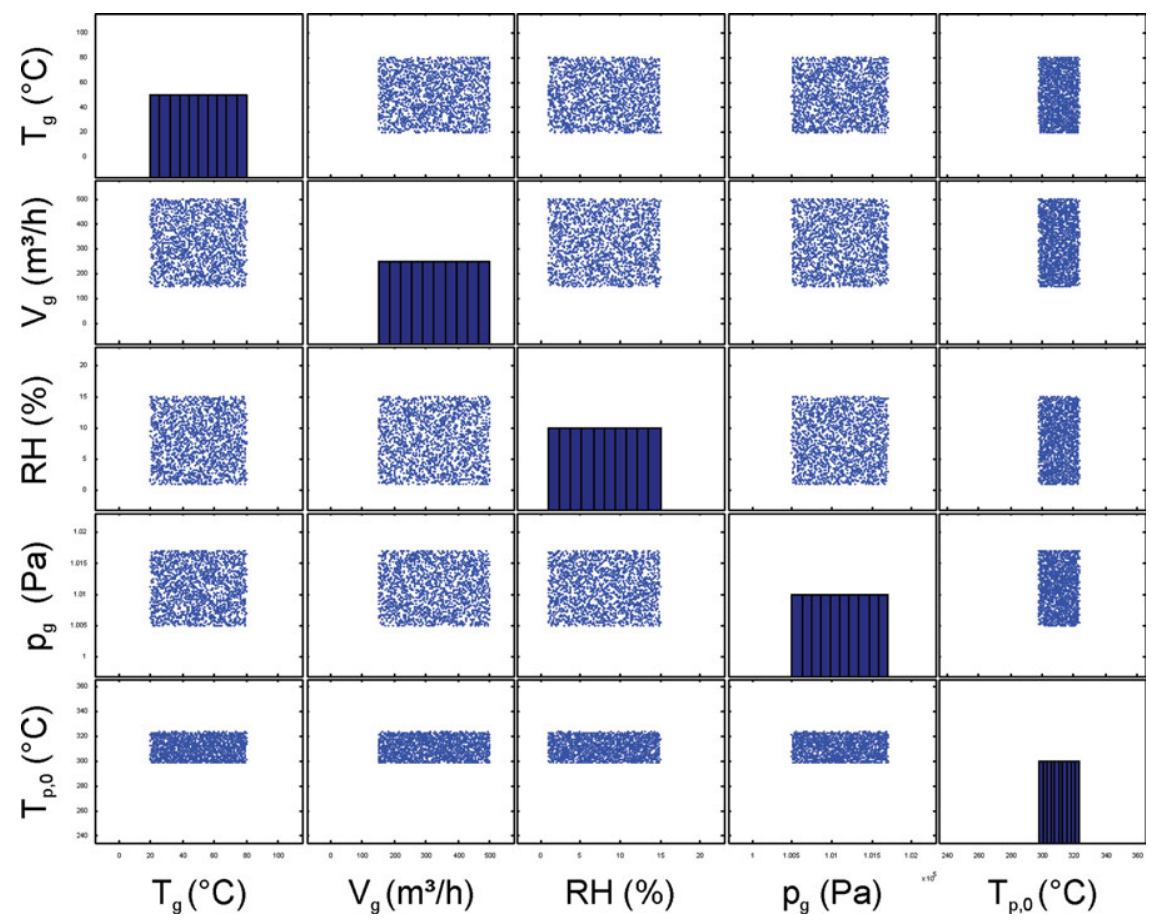

Figure 2. Distribution of the degrees of freedom resulting from applying Latin Hypercube Sampling.

[Color figure can be viewed in the online issue, which is available at wileyonlinelibrary.com.] 

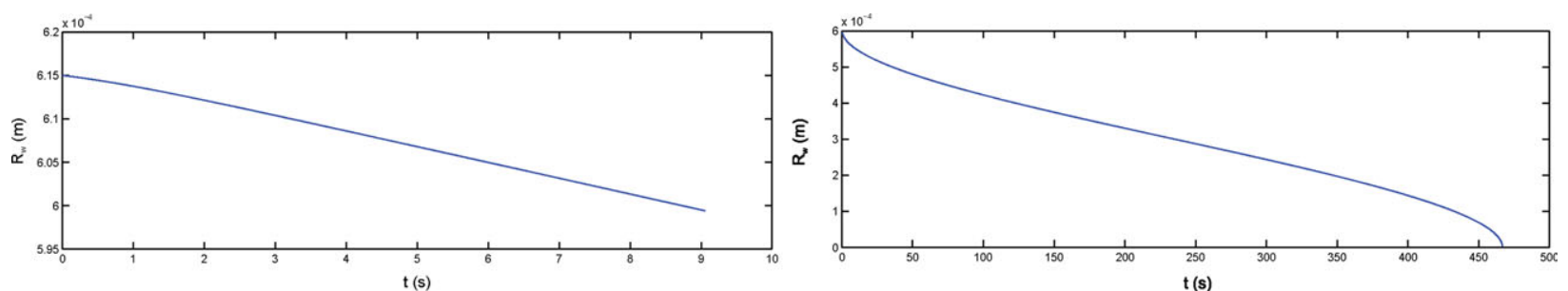

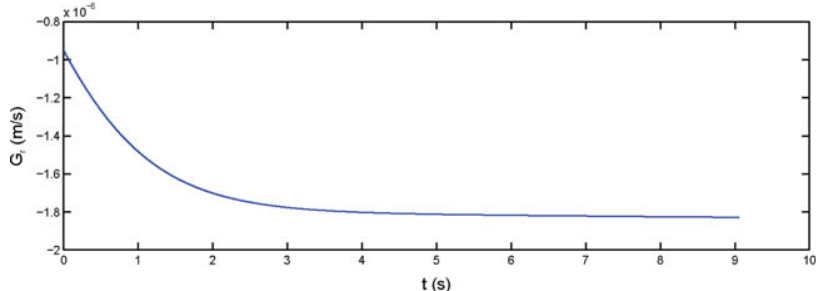

(a) Drying phase 1

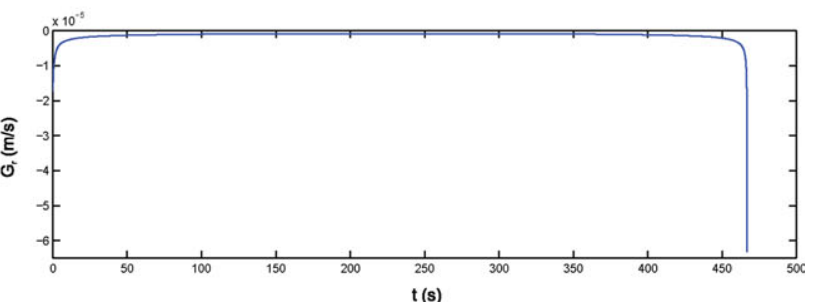

(b) Drying phase 2

Figure 3. Decrease in $\boldsymbol{R}_{\mathrm{w}}$ (top) and resulting growth term (bottom; $-55^{\circ} \mathrm{C}$ ).

[Color figure can be viewed in the online issue, which is available at wileyonlinelibrary.com.]

model $(g)$, which is function of variables, the selected degrees of freedom $(D)$, and the determined coefficients $(C)$.

\section{GSA}

The distribution of the degrees of freedom, generated by a uniform Latin Hypercube Sampling technique, is presented in Figure 2. The degrees of freedom are distributed uniformly in the parameter space, which can be seen clearly in the scatter plots. On the diagonal, histograms of the different degrees of freedom used in the GSA are presented. The other plots are normal scatter plots, and it is clear that the whole parameter space is explored by the Latin Hypercube Sampling technique.

Each set of degrees of freedom was evaluated and the growth term for the first and the second drying phase were calculated. Figure 3 shows the decrease in $R_{\mathrm{w}}$ as a function of time (top), as well as the resulting growth term (bottom). The first drying period is characterized by a growth term that shows an increase in negative rate at the start (i.e. increased drying rate) but levels off to a constant value near the end of the phase (Figure 3a). The absolute value of the growth term for the second drying period decreased strongly (becoming less negative, meaning drying at a slower rate) in the beginning, but after reaching a minimum rate, it increased again (Figure $3 b$ ). It can be concluded that the dynamics of both drying phases are clearly different.

In Figure 4, the growth term is plotted as a function of time for all different sets of degrees of freedom in the Monte Carlo analysis. It is obvious that the chosen combination of degrees of freedom for the sensitivity analysis has an influence on the drying time and behavior.

The black vertical line crossing the different simulations in Figure 4a marks the time point that is used for the linear regression. Results of simulations at this point are scaled and used in a least squares linear regression. As the drying model does not reach steady state, the alternative is that the output of the model has to be compared after a certain time step. Another choice would have been to take the average of all outputs for one simulation and to perform the linear regression with those averaged output values. The disadvantage of this approach is that the $R^{2}$ can become too low, so no conclusion can be made about the sensitivity of the degrees of freedom. In the first drying phase, the linear regression was performed after 3 s, where an $R^{2}$ of 0.97 was obtained. The second drying phase is longer, and therefore two timesteps were chosen

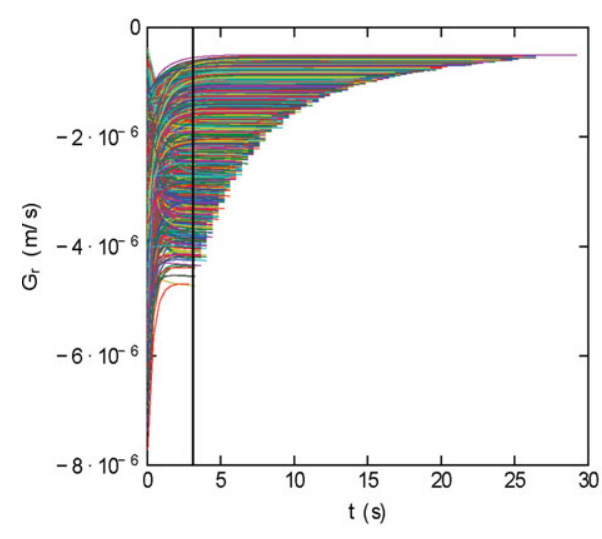

(a) Drying phase 1

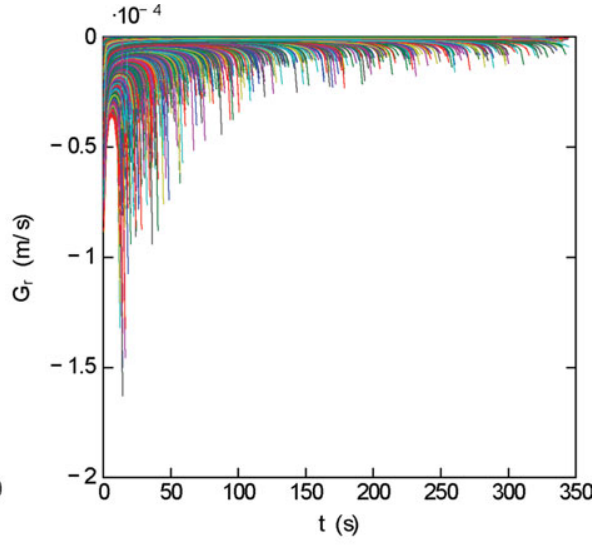

(b) Drying phase 2

Figure 4. The growth term for different parameter sets.

[Color figure can be viewed in the online issue, which is available at wileyonlinelibrary.com.] 
Table 2. Results of the GSA: SRCs for the Different Degrees of Freedom

\begin{tabular}{|c|c|c|c|}
\hline \multirow[b]{3}{*}{ Degree of Freedom } & \multicolumn{3}{|c|}{ Drying Phase } \\
\hline & \multirow{2}{*}{$\frac{\text { First }}{3 \mathrm{~s}}$} & \multicolumn{2}{|c|}{ Second } \\
\hline & & $1 \mathrm{~s}$ & $11 \mathrm{~s}$ \\
\hline$T_{\mathrm{g}}$ & 0.93 & 0.87 & 0.73 \\
\hline$V_{\mathrm{g}}$ & 0.29 & 0.02 & 0.03 \\
\hline $\mathrm{RH}$ & 0.13 & 0.02 & 0.05 \\
\hline$p_{\mathrm{g}}$ & 0.00 & 0.00 & 0.01 \\
\hline$T_{\mathrm{p}, 0}$ & 0.02 & 0.03 & 0.03 \\
\hline$R^{2}$ & 0.97 & 0.75 & 0.57 \\
\hline
\end{tabular}

for the linear regression, namely, 1 and $11 \mathrm{~s}$, and a clear difference in $R^{2}$ can be seen (Table 2). An $R^{2}$ of 0.57 is too low to draw conclusions, because normally a minimum of 0.7 is assumed for $R^{2}$.

The SRCs for the linear regression were calculated (Table 2). The ranking can be used to detect the most sensitive degrees of freedom. In both drying periods, the gas temperature clearly comes out as being the most sensitive degree of freedom. For the first drying phase, the gas velocity is also a quite sensitive degree of freedom, followed by the humidity. For the second drying phase, a different ranking can be observed when comparing evaluation after 1 or $11 \mathrm{~s}$. Because the gas temperature is clearly the most sensitive degree of freedom in both drying phases, the drying model was reduced using this degree of freedom.

\section{Model reduction}

The reduced model should be able to describe the decrease of the moisture content for a population of drying particles when incorporated in a PBE. Analysis of Figure 3

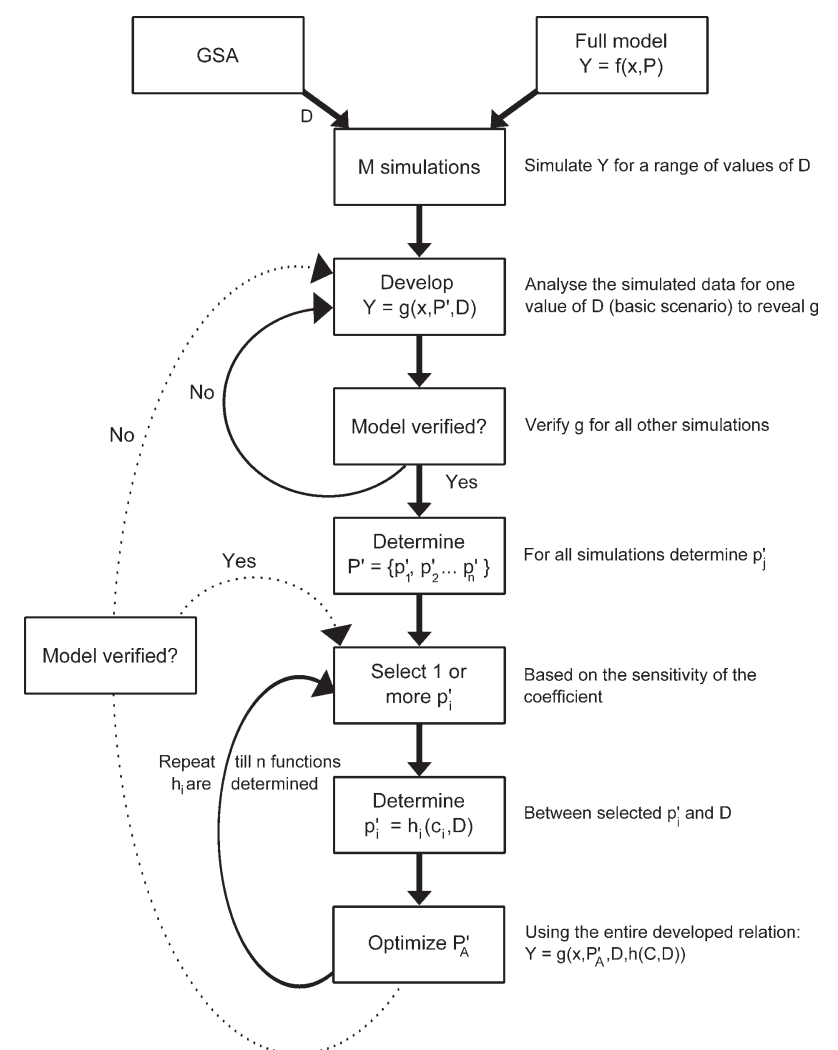

Figure 5. Scheme of the steps taken during the model reduction procedure.

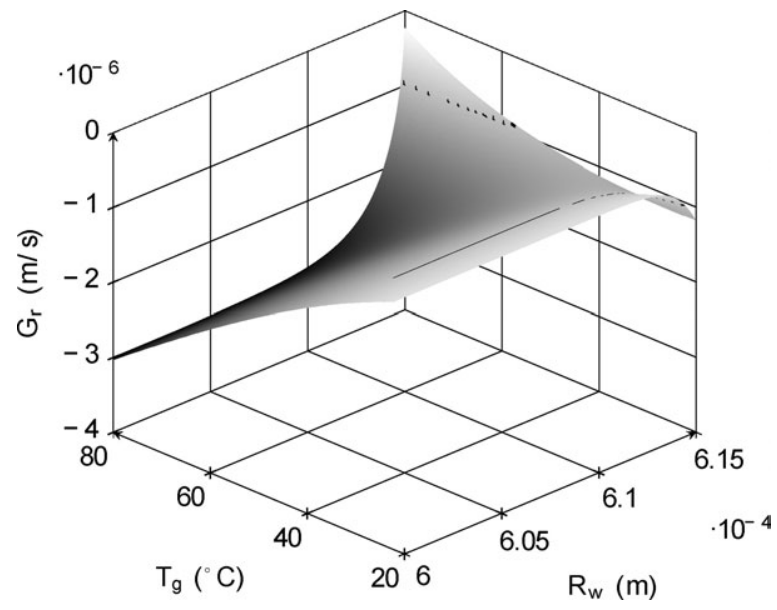

Figure 6. Growth term in function of the wet radius for different gas temperatures for the first drying phase.

revealed that the growth term is strongly dependent on the radius of the particle (first drying phase) and the radius of the wet core (second drying phase). It was, therefore, decided to develop a reduced model in function of this radius, which means a size dependent growth term will be used in the PBM model (Eq. 4). Based on the GSA, the gas temperature was chosen as the most sensitive degree of freedom. The reduced model, function of the most sensitive degrees of freedom only, implemented in the PBE should be able to compute the evolution of the moisture content of a population of particles that are drying, all subjected to the same ambient conditions.

The general procedure followed during the model reduction is presented in Figure 5. More details about the elaboration of the method are described in the Appendices, where the procedure is worked out in detail for our specific case study.

As mentioned in previous sections, a GSA was performed to detect the most sensitive degrees of freedom. Simulations are performed while varying the selected degrees of freedom $(D)$ in a range between a minimum and a maximum value. $M$ is the number of simulations that are performed. The range is based on the physical limitations of the dryer and the physical reality as for the GSA. The most obvious simulation, which is situated in the middle of the range or is physically most frequently used, is chosen as basic scenario to develop a model structure $(g)$ with $n$ coefficients. The model structure is verified for the other simulations, and afterward the coefficients $\left(P^{\prime}\right)$ are optimized by minimizing the Root Mean Squared Error (RMSE) between the simulated data and the predictions of the developed model structure for each value of the selected degrees of freedom in the range. This results in a matrix with $n$ rows and $M$ columns

$$
P^{\prime}=\left(\begin{array}{ccccc}
p_{1,1}^{\prime} & p_{1,2}^{\prime} & \cdots & p_{1, M-1}^{\prime} & p_{1, M}^{\prime} \\
p_{n, 1}^{\prime} & p_{n, 2}^{\prime} & \cdots & p_{n, M-1}^{\prime} & p_{n, M}^{\prime}
\end{array}\right)
$$

with $p_{i, j}^{\prime}$ the parameter value corresponding to the $i$ th coefficient and $j$ th simulation. $p_{i}^{\prime}$ is a vector with the values for coefficient $i$ for all simulations, whereas $p_{j}^{\prime}$ is a vector with all coefficients for one simulation.

The optimized values of the coefficients are plotted in function of the degree of freedom. One or more coefficients 


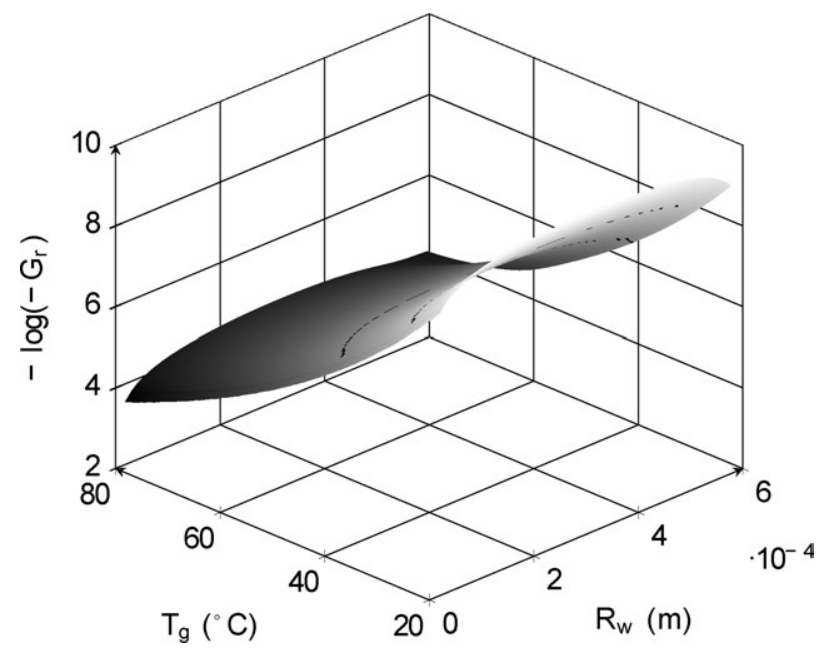

Figure 7. Growth term in function of the wet radius for different gas temperatures for the second drying phase.

are selected, and a relation $\left(h_{i}\right)$ is determined between $p_{i}^{\prime}$ and $D$ with $c_{i}$ the introduced coefficients. The sensitivity of the coefficients $\left(p_{i}^{\prime}\right)$ or the absence of noise can help to select these first coefficients. The relation can be a polynomial where the order is dependent on the desired accuracy. Afterward, the relation $h_{i}$ is implemented as fixed in the developed model structure, and the other coefficients $\left(P_{A}^{\prime}\right)$ are optimized again. As such a deviation in the relation with respect to the fitted coefficient can be caught. At certain steps in the procedure, the global model structure is verified again. These last steps are repeated till all relations $\left(h_{1}\right.$ till $h_{n}$ ) between the coefficients $\left(P^{\prime}\right)$ and the selected degrees of freedom $(D)$ are determined. At the end, a global optimization can be performed to optimize the values of all coefficients $(C)$ of the reduced model simultaneously.

The drying model was simulated for gas temperatures ranging from 20 to $80{ }^{\circ} \mathrm{C}$ with a particle radius of $0.6 \mathrm{~mm}$. For both drying phases, the growth term, that is, the derivative of $R_{\mathrm{w}}$ (Eq. 18), was calculated numerically with a resolution $(\Delta t)$ of $0.2 \mathrm{~s}$ and plotted against the wet radius, $R_{\mathrm{w}}$ (Figures 6 and 7).

$$
\dot{R_{\mathrm{W}}}=G_{\mathrm{r}, 1}\left(R_{\mathrm{w}, \text { nor }}, T_{\mathrm{g}}\right) \cong \frac{R_{\mathrm{w}}\left(t_{i+1}\right)-R_{\mathrm{w}}\left(t_{i}\right)}{\Delta t}
$$

It is obvious that a different model structure will be required for both drying phases. The objective is the development of a simpler model able to describe the behavior visualized in Figures 6 and 7.

Table 3. Resulting Equations for the Different Coefficients of the First Drying Phase

\begin{tabular}{llcl}
\hline Coefficient & Polynomial function \\
\hline$A$ & $=$ & $a_{1} \cdot T_{\mathrm{g}}^{4}+a_{2} \cdot T_{\mathrm{g}}^{3}+a_{3} \cdot T_{\mathrm{g}}^{2}+a_{4} \cdot T_{\mathrm{g}}+a_{5}$ & $(23)$ \\
$B$ & $=$ & $b_{1} \cdot T_{\mathrm{g}}^{3}+b_{2} \cdot T_{\mathrm{g}}^{2}+b_{3} \cdot T_{\mathrm{g}}+b_{4}$ \\
$C$ & $=$ & $c_{1} \cdot \exp \left(-\left(T_{\mathrm{g}}+c_{2}\right)^{2} / c_{3}\right)$ \\
& & $+c_{4} \cdot \exp \left(-\left(T_{\mathrm{g}}+c_{5}\right)^{2} / c_{6}\right)$ \\
$D$ & $=$ & $d_{1} \cdot T_{\mathrm{g}}^{2}+d_{2} \cdot T_{\mathrm{g}}+d_{3}$
\end{tabular}

Table 4. Resulting Equations for the Different Coefficients for the Second Drying Phase

\begin{tabular}{llc}
\hline Coefficient & \multicolumn{2}{c}{ Describing function } \\
\hline$A^{\prime}$ & $=$ & $-\exp \left(a_{1}^{\prime}\right) \cdot T_{\mathrm{g}}^{a_{2}^{\prime}}$ \\
$B^{\prime}$ & $=$ & $b_{1}^{\prime} \cdot \exp \left(b_{2}^{\prime} \cdot\left(T_{\mathrm{g}}-20\right)\right)-1 \quad(28)$ \\
$C^{\prime}$ & $-\exp \left(c_{1}^{\prime}\right) \cdot T_{\mathrm{g}}^{c_{2}^{\prime}}$ & $(29)$ \\
$D^{\prime}$ & $=$ & $\exp \left(d_{1}^{\prime}\right) \cdot \exp \left(d_{2}^{\prime} \cdot T_{\mathrm{g}}\right)-1 \quad(30)$ \\
$E^{\prime}$ & $=$ & $e_{1}^{\prime} \cdot \exp \left(e_{2}^{\prime} \cdot\left(T_{\mathrm{g}}-20\right)\right)-1 \quad(31)$ \\
$R_{f}^{\prime}$ & $=$ & $R_{\mathrm{f}, 1}^{\prime} \cdot T_{\mathrm{g}}^{\prime R_{\mathrm{f}, 2}^{\prime}}+R_{\mathrm{f}, 3}^{\prime}$ \\
\hline
\end{tabular}

The developed model structure for the first drying phase is

$$
\begin{gathered}
G_{\mathrm{r}, 1}\left(R_{\mathrm{w}, \text { nor }}, T_{\mathrm{g}}\right)=A+B \cdot R_{\mathrm{w}, \text { nor }}+C \cdot \exp ^{D \cdot R_{\mathrm{w}, \text { nor }}} \\
R_{\mathrm{w}, \text { nor }}=\frac{R_{\mathrm{w}}-R_{\mathrm{p}}}{R_{\mathrm{w}, 0}-R_{\mathrm{p}}}
\end{gathered}
$$

with $A, B, C$, and $D$ empirical coefficients, $R_{\mathrm{p}}$ the radius of the dry particle, and $R_{\mathrm{w}, 0}$ the initial (wet) radius. The equations for the different coefficients of the first drying phase are mentioned in Table 3. More details about the used procedure are mentioned in Appendix A.

The global empirical equation for the first drying phase exhibits a mean weighted relative error of $0.53 \%$ between the simulated detailed drying model prediction and the empirical model.

For the second drying phase the resulting equation is

$$
\begin{gathered}
G_{\mathrm{r}, 2}\left(R_{\mathrm{w}, \text { nor }}^{\prime}, T_{\mathrm{g}}\right)=A^{\prime} \cdot\left(R_{\mathrm{w}, \text { nor }}^{\prime}\right)^{B^{\prime}}+C^{\prime} \cdot\left(1+D^{\prime} \cdot R_{\mathrm{w}, \text { nor }}^{\prime}\right)^{E^{\prime}} \\
+R_{\mathrm{f}}^{\prime} *\left(A^{\prime} \cdot 0.5^{B^{\prime}}+C^{\prime} \cdot\left(1+D^{\prime} \cdot 0.5\right)^{E^{\prime}}\right) \\
R_{\mathrm{w}, \text { nor }}^{\prime}=\frac{R_{\mathrm{w}}}{R_{\mathrm{p}}}
\end{gathered}
$$

with $A^{\prime}, B^{\prime}, C^{\prime}, D^{\prime}$, and $E^{\prime}$ empirical coefficients and $R_{\mathrm{f}}^{\prime}$ a factor to reduce the offset. In Table 4 , the equations for the different coefficients are mentioned for the second drying phase. Details about the procedure can be found in Appendix B.

The global equation for the second drying period has a mean weighted relative error of $1.97 \%$ between the full model result and the empirical fit.

Table 5. Parameter Values for the First Drying Phase

\begin{tabular}{ll}
\hline$a_{1}$ & $-6.43 \mathrm{e}-15$ \\
$a_{2}$ & $-2.74 \mathrm{e}-12$ \\
$a_{3}$ & $-5.28 \mathrm{e}-10$ \\
$a_{4}$ & $-7.24 \mathrm{e}-09$ \\
$a_{5}$ & $-2.35 \mathrm{e}-07$ \\
$b_{1}$ & $1.45 \mathrm{e}-12$ \\
$b_{2}$ & $-1.30 \mathrm{e}-10$ \\
$b_{3}$ & $-3.99 \mathrm{e}-09$ \\
$b_{4}$ & $-2.57 \mathrm{e}-08$ \\
$c_{1}$ & $4.97 \mathrm{e}-12$ \\
$c_{2}$ & 62.4 \\
$c_{3}$ & 202.82 \\
$c_{4}$ & $-3.05 \mathrm{e}-13$ \\
$c_{5}$ & 36.43 \\
$c_{6}$ & 96.14 \\
$d_{1}$ & 0.0037 \\
$d_{2}$ & -0.408 \\
$d_{3}$ & 23.5 \\
\hline
\end{tabular}


Table 6. Parameter Values for the Second Drying Phase

\begin{tabular}{lc}
\hline$a_{1}^{\prime}$ & 9.93 \\
$a_{2}^{\prime}$ & -55.1 \\
$b_{1}^{\prime}$ & -0.130 \\
$b_{2}^{\prime}$ & -0.381 \\
$c_{1}^{\prime}$ & 10.3 \\
$c_{2}^{\prime}$ & -56.7 \\
$d_{1}^{\prime}$ & 0.0995 \\
$d_{2}^{\prime}$ & -10.0 \\
$e_{1}^{\prime}$ & -0.0998 \\
$e_{2}^{\prime}$ & -0.486 \\
$R_{\mathrm{f}, 1}^{\prime}$ & $7.24 \mathrm{e} 04$ \\
$R_{\mathrm{f}}^{\prime}, 2$ & -3.51 \\
$R_{\mathrm{f}, 3}^{\prime}$ & -0.113 \\
\hline
\end{tabular}

The resulting optimized values for the parameters of the reduced model for both drying phases are tabulated in Tables 5 and 6 , respectively.

\section{PBM}

The developed reduced drying model was intended for use in a PBE. The developed reduced model, a quite straightforward combination of algebraic equations, can be easily implemented in different solution methods for PBM, which was not possible for the original drying model. In this case, the PBM was solved using HRFV. ${ }^{6}$ The initial distribution used is a normal probability density distribution with standard deviation $(\sigma)$ of $3 \mathrm{e}-6$ and mean $(\mu)$ of 1.02 times $R_{\mathrm{p}}$, which is a realistic condition for a population of granules originating from a granulator. The simulation with the PBE is done at a fixed temperature of $55{ }^{\circ} \mathrm{C}$. The evolution of the number density in time is presented in Figure 8. As time evolves, the width of the number density distribution increases which means that the drying rate of different granules is different, and results in a broader distribution of moisture content of the population. The evolution of $R_{\mathrm{w}}$, which represent the moisture content of a wet particle, is in line with the expectations obtained with the full drying model. Similar simulations can now be run for different gas temperatures, which indicate the impact on the drying process. In Figure 9, the number density after 20 and $40 \mathrm{~s}$ is presented for different gas temperatures, starting from the same initial condition. It is clear that the decrease of the moisture content is faster for higher gas temperatures.

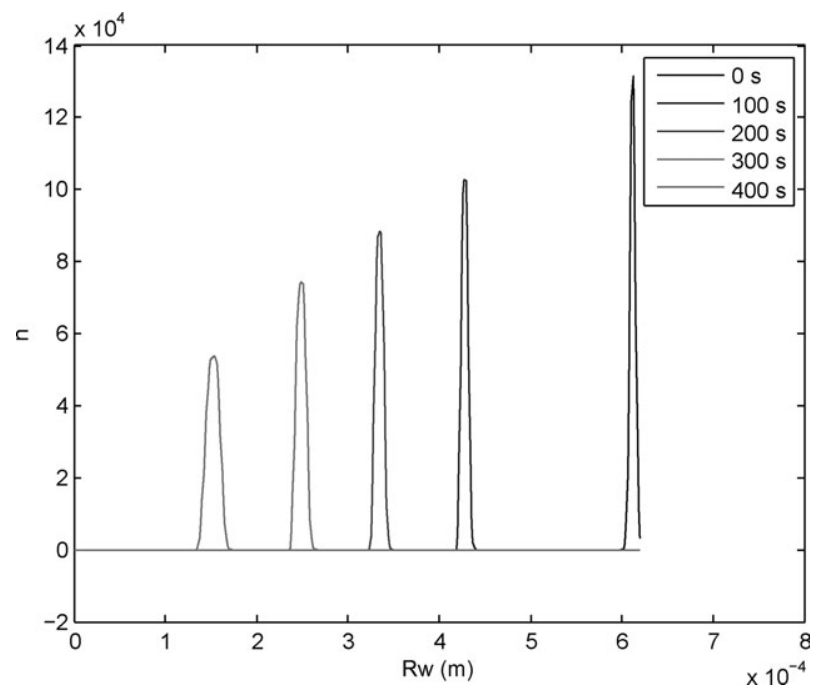

Figure 8. Evolution of number density using PBM at $55^{\circ} \mathrm{C}$.

Finally, also the initial moisture content can be varied. This is beyond the scope of this article and will be investigated in a future contribution.

\section{Discussion}

The procedure mentioned in this article has clearly advantages and disadvantages. It is a heuristic reduction technique, but as stated by Van Nes et al. ${ }^{21}$ it is very powerful, if the complex and the reduced model produces similar results. ${ }^{21}$ To start the model reduction procedure, knowledge about the process is required. Here, the big difference in drying behavior of the first and second drying phase was known a priori, and this knowledge was used to decide to make a separate reduced model for both phases. A GSA was used to detect the most influential input variables but also insight into the process, and the model was helpful to understand why these variables were important. If two input variables were equally influential, a choice was made based on the expected range in the dryer and experience with the process under consideration. The pitfall of model reduction techniques described in the literature can be that the result is a reduced model that is too complex for the purpose. The described approach has the advantage of allowing full control over the reduction process

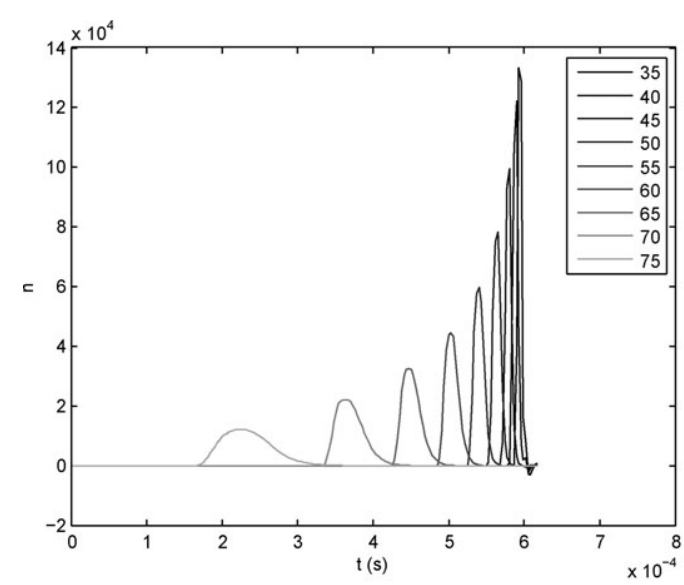

(a) $20 \mathrm{~s}$

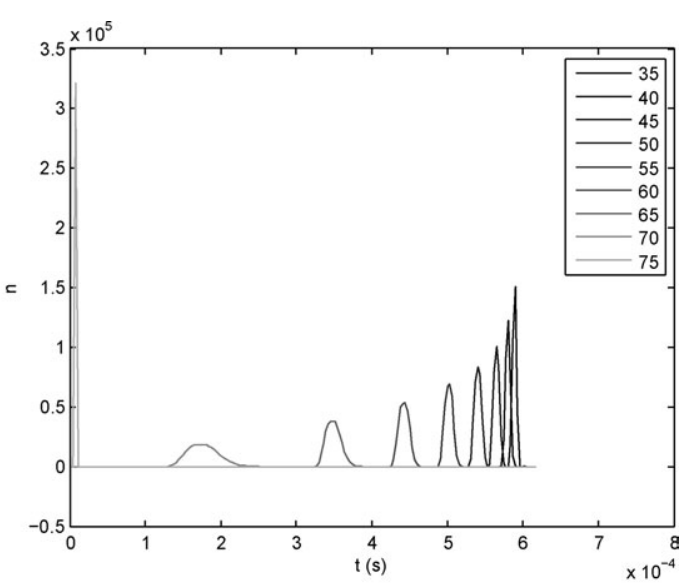

(b) $40 \mathrm{~s}$

Figure 9. Number density for different gas temperatures at different time steps. 
in the sense that the complexity of the empirical model to be obtained can be chosen. This will be a trade-off with the loss of accuracy (i.e., the relative error between the physical model and the reduced empirical model). In the derived model here, complexity of the model was not penalized as it was no restriction for the subsequent step (see Section PBM). The resulting reduced model contains the input variable that is first of all the most sensitive variable but is also a variable that is important in further research. The reduced model will be used in a PBM model, and at a later stage the PBM model can be extended with the information of a CFD model of the dryer. The disadvantage of choosing the equations of the reduced model arbitrarily lies in the fact that these equations are not necessarily the best equations to describe the drying behavior. There is always a chance that more simple equations are sufficient. On the other hand, the choice of the equations can be made in function of the purpose, in this case the implementation into a PBM model and maybe later in a coupled PBM-CFD model.

\section{General Conclusion}

Starting from a complex model describing the drying behavior of individual granules, it was possible to develop a reduced model that can now be used as submodel in other models, for example, a PBM. A new strategy to perform a model reduction was introduced and demonstrated. The development of the reduced model started with a GSA to detect the most sensitive degrees of freedom in the model. The full drying model was simulated for a range of values of the most sensitive degree of freedom to have some data to use for the development of the reduced model. Based on the data, a proposal for an empirical model is made, which can be reviewed in a later stage of the model reduction procedure. The coefficients of the proposed reduced model are described in function of the most sensitive degree of freedom. The reduced model is an empirical model able to calculate the decrease in $R_{\mathrm{w}}$ for different gas temperatures regardless the granule radius. The developed procedure is able to construct a reduced model with a low mean weighted relative error.

The empirical model can now be used to further analyze the drying behavior of pharmaceutical granules in a fluidized bed dryer. The objective is to study the properties of a population of particles and the evolution of the moisture content in the dryer. To fully understand their behavior, the developed PBM model should be combined with CFD model to take into account local variations of temperature and humidity in the dryer and their effect on the drying process.

\section{Acknowledgment}

Financial support for this research from the Fund for Scientific Research Flanders (FWO Flanders-Ph.D. fellowship Séverine Thérèse F.C. Mortier) is gratefully acknowledged.

\section{Literature Cited}

1. Hegedus A, Pintye-Hódi K. Comparison of the effects of different drying techniques on properties of granules and tablets made on a production scale. Int J Pharm. 2007;330:99-104.

2. Mortier STFC, De Beer T, Gernaey KV, Vercruysse J, Fonteyne M, Remon JP, Vervaet C, Nopens I. Mechanistic modelling of the drying behaviour of single pharmaceutical granules. Eur J Pharm Biopharm. 2012;80:682-689.

3. Mortier STFC, De Beer T, Gernaey KV, Remon JP, Vervaet C, Nopens I. Mechanistic modelling of fluidized bed drying processes of wet porous granules: a review. Eur $J$ Pharm Biopharm. 2011;79:205-225.

4. Ramkrishna D. Population Balances: Theory and Applications to Particulate Systems in Engineering. Academic Press, New York. 2000.

5. Mesbah A, Kramer HJM, Huesman AEM, Van Den Hof PMJ. A control oriented study on the numerical solution of the population balance equation for crystallization processes. Chem Eng Sci. 2009;64:4262-4277.

6. Qamar S, Elsner M, Angelov I, Warnecke G, Seidel-Morgenstern A. A comparative study of high resolution schemes for solving population balances in crystallization. Comput Chem Eng. 2006;30:11191131.

7. Marchisio DL, Pikturna JT, Fox RO, Vigil RD, Barresi AA. Quadrature method of moments for population-balance equations. AIChE J. 2003;49:1266-1276.

8. Qamar S, Warnecke G. Numerical solution of population balance equations for nucleation, growth and aggregation processes. Comput Chem Eng. 2007;31:1576-1589.

9. Antoulas AC. An overview of approximation methods for large-scale dynamical systems. Ann Rev Control. 2005;29:181-190.

10. Banerjee S, Vernon Cole J, Jensen KF. Nonlinear model reduction strategies for rapid thermal processing systems. IEEE Trans Semicond Manuf. 1998;11:266-275.

11. Van Nes EH, Scheffer M. A strategy to improve the contribution of complex simulation models to ecological theory. Ecol Model. 2005; 185:153-164.

12. Bernhardt K. Finding alternatives and reduced formulations for process-based models. Evol Comput. 2008;16:63-88.

13. Phillips J. Projection frameworks for model reduction of weakly nonlinear systems. In: Proceedings of the 37th Design Automation Conference, 2000:184-189.

14. Degenring D, Froemel C, Dikta G, Takors R. Sensitivity analysis for the reduction of complex metabolism models. J Process Contr. 2004; 14:729-745.

15. Lawrie J, Hearne J. Reducing model complexity via output sensitivity. Ecol Model. 2007;207:137-144.

16. Saltelli A, Tarantola S, Campolongo F, Ratto M. Sensitivity Analysis in Practice: A Guide to Assessing Scientific Models. Chichester, UK: Wiley, 2004.

17. McKay MD, Beckman RJ, Conover WJ. A comparison of three methods for selecting values of input variables in the analysis of output from computer code. Technometrics. 2010;42:55-61.

18. Saltelli A, Ratto M, Andres T, Campolongo F, Cariboni J, Gatelli D, Saisana M, Tarantola S. Global Sensitivity Analysis The Primer. Chichester, UK: Wiley, 2008.

19. Saltelli A. The critique of modelling and sensitivity analysis in the scientific discourse: an overview of good practices. In TAUC, Washington, 2006.

20. Chahlaoui Y, Lemonnier D, Vandendorpe A, Van Dooren PM. Second order balanced truncation. Linear Algebra Appl. 2005;415:373384.

21. Van Nes EH, Scheffer M, Van den Berg MS, Coops H. Aquatic macrophytes: restore, adicate or is there a compromise? Aquat Bot. 2002;72:387-403.

\section{Appendix A}

For the first drying phase, the curves display an exponential behavior at fixed gas temperatures (Figure 3a). The data obtained at a gas temperature of $55^{\circ} \mathrm{C}$ were used as a starting point (basic scenario). The nonlinear behavior on a logscale suggested that also other terms should be taken into account. A pure exponential behavior results in a linear equation on a log-scale.

$$
\begin{gathered}
y=a \mathrm{e}^{b x} \\
\log (y)=\log (a)+\log (e) b x
\end{gathered}
$$

First, $R_{\mathrm{w}}$ was normed to simplify the adaptation of the reduced model to other particle sizes and initial moisture content (Eq. 20), after which the first-and higher-order derivatives of the simulated $\mathrm{G}_{\mathrm{r}, 1}$ with respect to $R_{\mathrm{w}}$ (same procedure as Eq. 18) were taken. The second-order derivative of 


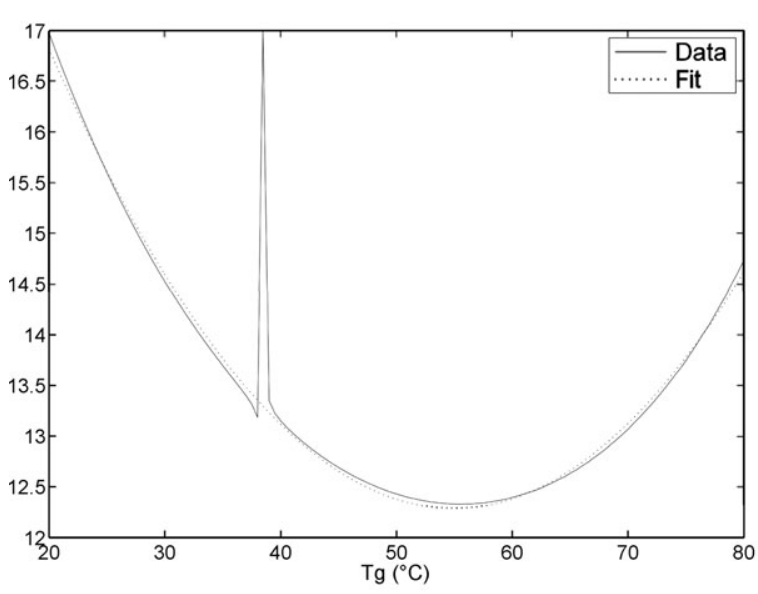

(a) $D$

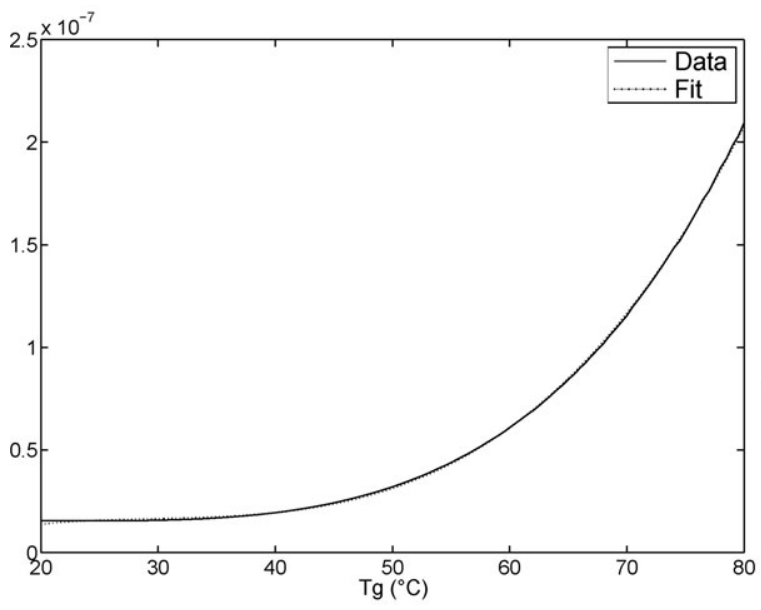

(c) $B$

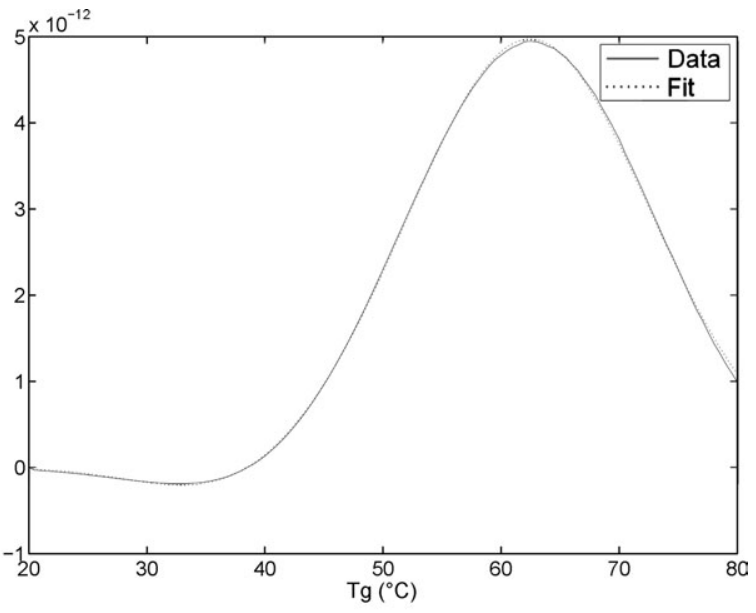

(b) $C$

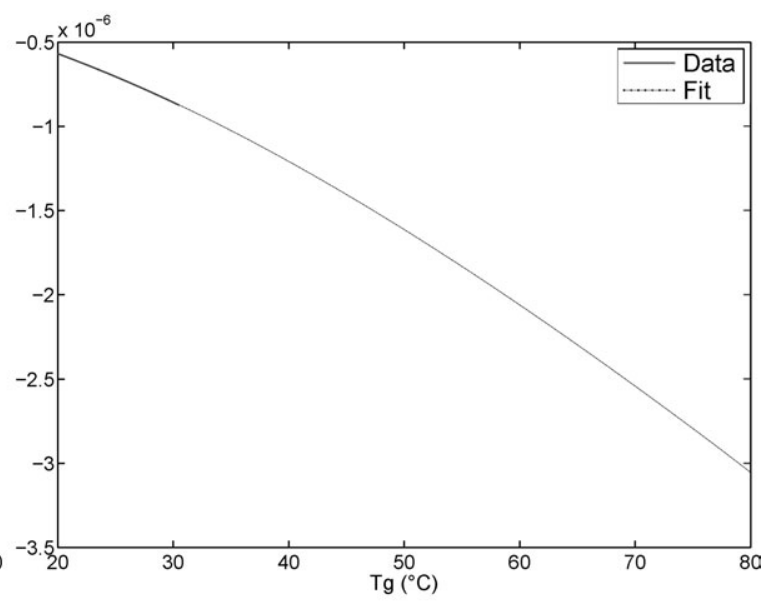

(d) $A$

Figure A1. Coefficients in function of the gas temperature ranging from 35 to $75^{\circ} \mathrm{C}$ for the first drying period.

the data showed a linear trend on a log-scale. Hence, the proposed expression for describing the growth term for the first drying phase was Eq. 19. A first estimate of the different coefficients was based on the first till third derivative of the growth function

$$
\begin{aligned}
& \frac{\mathrm{d} G_{\mathrm{r}, 1}\left(R_{\mathrm{w}, \text { nor }}\right)}{\mathrm{d} R_{\mathrm{w}, \text { nor }}}=B+C \cdot D \cdot \mathrm{e}^{D \cdot R_{\mathrm{w}, \text { nor }}} \\
& \frac{\mathrm{d}^{2} G_{\mathrm{r}, 1}\left(R_{\mathrm{w}, \text { nor }}\right)}{\mathrm{d} R_{\mathrm{w}, \text { nor }}^{2}}=C \cdot D^{2} \cdot \mathrm{e}^{D \cdot R_{\mathrm{w}, \text { nor }}} \\
& \frac{\mathrm{d}^{3} G_{\mathrm{r}, 1}\left(R_{\mathrm{w}, \text { nor }}\right)}{\mathrm{d} R_{\mathrm{w}, \text { nor }}^{3}}=C \cdot D^{3} \cdot \mathrm{e}^{D \cdot R_{\mathrm{w}, \text { nor }}}
\end{aligned}
$$

Using this approach coefficient $D$ could be obtained for each simulation at a different gas temperature by dividing Eq. A3-c by Eq. A3-b. Once $D$ is determined, a step-wise approach can be used to obtain estimates of the other coefficients. $C$ could be easily determined out of Eq. A3-b or A3-c. Afterward, coefficient $B$ could be obtained by calculating the offset between the first derivative and Eq. A3-a. The same procedure was followed for determining coefficient $A$, but then it was performed by comparing the original data of the growth with Eq. 19. Subsequently, the values of the obtained coeffi- cients were optimized using an optimization algorithm (i.e., fminsearchbnd3 from Matlab $\left.^{\circledR}\right)$. This procedure can be repeated for all gas temperatures. Finally, coefficient $D$ was determined in function of the gas temperature using a polynomial fit. The order of the polynomial was fixed at 2 . The higher the order, the more accurate the polynomial will describe the behavior, but the more parameters will be introduced. Some outliers were detected (numerical errors resulting from the computation of derivatives) and removed, after which the polynomial fit was repeated for the smoothed data. The resulting polynomial is shown in Figure A1a (Eq. 26). The reason for the peak in the curve is that the value at this point has no influence as coefficient $C$ is zero at this point.

Using the fixed relation between coefficient $D$ and the gas temperature, the other coefficients were optimized again. This was done, because a deviation in the polynomial with respect to the fitted coefficient can be caught by this. The relation between $D$ and $T_{\mathrm{g}}$ is a fit and will not give exactly the correct value for each $T_{\mathrm{g}}$. To make sure the final reduced model is able to calculate the growth term as reliable as possible, the coefficients are optimized again by minimizing the error between the simulated result and the reduced model (using fminsearchbnd3 from Matlab ${ }^{\circledR}$ ). Afterward, a relation was looked for between coefficient $C$ and the gas temperature (Figure A1b; Eq. 25). 
In a similar step-wise fashion, coefficients $B$ and $A$ were optimized and a polynomial was fit to relate the optimized value and the gas temperature (Figures A1-c,d) (Eqs. 24 and 23 , respectively). As the polynomial for $A$ was determined in the final step, the order was chosen somewhat higher.

The resulting gas temperature dependent functions are summarized in Table 3, where $T_{g}$ represents the gas temperature in ${ }^{\circ} \mathrm{C}$.

\section{Appendix B}

The behavior of the second drying phase is different (Figure 7). Again, the data at a gas temperature of $55{ }^{\circ} \mathrm{C}$ were used for the basic scenario. The growth of the second drying phase showed a symmetric shape around a normalized radius $R_{\mathrm{w}, \mathrm{nor}}^{\prime}$ of 0.5 . Therefore, it was hypothesized to use the same function for both parts, which could be easily added together. The curve also showed an infinite behavior when $R_{\mathrm{w}, \text { nor }}^{\prime}$ was approaching zero or one. Therefore, the most convincing hypothesis was to use a generalized hyperbolic function $\left(A^{\prime} \cdot R_{\mathrm{w}, \text { nor }}^{\prime}{ }^{\prime}\right)$ for describing the curve for low values of $R_{\mathrm{w}, \mathrm{nor}}^{\prime}$. For values higher than a normalized radius of a half a similar equation was used to fit the data, namely $C^{\prime} \cdot\left(1+D^{\prime} \cdot R_{\mathrm{w}, \mathrm{nor}}^{\prime}\right)^{E^{\prime}}$. By combining the previous functions, the data could be described in an appropriate way

$$
G_{\mathrm{r}, 2}\left(R_{\mathrm{w}, \mathrm{nor}}^{\prime}, T_{\mathrm{g}}\right)=A^{\prime} \cdot\left(R_{\mathrm{w}, \mathrm{nor}}^{\prime}\right)^{B^{\prime}}+C^{\prime} \cdot\left(1+D^{\prime} \cdot R_{\mathrm{w}, \mathrm{nor}}^{\prime}\right)^{E^{\prime}}
$$

Using all simulated data (at the different gas temperatures) and testing global optimization algorithms for all coeffi- cients, the obtained curves of the coefficients in function of the gas temperature were not smooth at all, but it could be concluded that coefficients $B^{\prime}$ and $E^{\prime}$ could be approached by -1 in a first trial. With this information, coefficients $A^{\prime}$, $C^{\prime}$, and $D^{\prime}$ were subsequently optimized (with fminsearchbnd3 from Matlab ${ }^{\circledR}$ ). First, the relation between $A^{\prime}$ and $C^{\prime}$ and the gas temperature was determined (Eqs. 27 and 29). Because these relations are always an approximation, coefficient $D^{\prime}$ is optimized, before its relation with the gas temperature is determined (Eq. 30). After this step, coefficients $a_{1}^{\prime}, a_{2}^{\prime}, c_{1}^{\prime}, c_{2}^{\prime}, d_{1}^{\prime}$, and $d_{2}^{\prime}$ were optimized simultaneously.

Using these relations for the coefficients, an offset was found between the simulated result and the empirical model. The maximum value of the growth function for different gas temperatures varied between $-1.181 \mathrm{e}-10$ and $-3.383 \mathrm{e}-5$. As a result of the large range of values, it is not possible to minimize directly the difference between the simulated data and the empirical model. Therefore, a relative adaptation was introduced to remove the offset, resulting in Eq. 21. $R_{f}^{\prime}$ was determined for each simulated data point and in a next step also fitted in function of the gas temperature (Eq. 32). Using the calibrated $R_{f}^{\prime}$ relation coefficients $a_{1}^{\prime}, a_{2}^{\prime}, c_{1}^{\prime}, c_{2}^{\prime}$, $d_{1}^{\prime}$, and $d_{2}^{\prime}$ were optimized again. In a next step, coefficients $B^{\prime}$ and $E^{\prime}$ were optimized and the relation between them and the gas temperature was determined (Eqs. 28 and 31). However, coefficient $B^{\prime}$ and $E^{\prime}$ display a strong correlation. Finally all coefficients were optimized. The resulting equations are given in Table 4.

Manuscript received May 17, 2012, and revision received July 22, 2012. 